\title{
Atomistic Simulation of Stresses in Growing Silicon Dioxide Films
}

\author{
Fedor V. Grigoriev *, Vladimir B. Sulimov and Alexander V. Tikhonravov \\ RCC M.V. Lomonosov MSU, 119991 Moscow, Russian; vs@dimonta.com (V.B.S); tikh@srcc.msu.ru (A.V.T.) \\ * Correspondence: fedor.grigoriev@gmail.com
}

Received: 24 January 2020; Accepted: 27 February 2020; Published: 29 February 2020

\begin{abstract}
Dependence of stress values in silicon dioxide films on its thickness in the initial stage of film growth was investigated using atomistic molecular dynamics simulation. It was shown that the stress in normally deposited films was compressive and varied slightly with growth of film thickness. The stress in the glancing angle deposited films was several times lower than the stress in the normally deposited films, and varied from compressive stress to tensile stress with increasing film thickness. An essential anisotropy of stress tensor components was revealed for the case of glancing angle deposition. The calculated stress values were in the interval of experimental data.
\end{abstract}

Keywords: thin films; glancing angle deposition; molecular dynamics; silicon dioxide films; stresses

\section{Introduction}

Mechanical stresses that arise in growing films, due to differences in the substrate and film properties, significantly affect the optical coating quality. These stresses result in the deformation of the substrate, which may be a problem in coating applications [1]. There are two types of stresses-tensile and compressive, having a positive and negative sign, respectively. The value of the stresses essentially depend on the film's material and deposition conditions [1,2].

An experimental investigation of the stress dependence on deposition conditions at the initial stage of film growth is still a challenge. On the other hand, stress values can be calculated directly from the results of an atomistic simulation through the pressure applied to the boundaries of the simulation box. So the application of the atomistic simulation to the investigation of the stresses in the deposited films seems promising.

The value of the angle between the direction of the incoming flow of atoms and the normal to the substrate surface (deposition angle or incidence angle) is one of the key parameters affecting the film properties. The near-normal deposition at a small value of incidence angle results in the formation of a dense and homogenous structure. In contrast, deposition with an incidence angle greater than approximately $60^{\circ}$ leads to the growth of anisotropic films with high porosity [3,4]. This deposition regime is termed glancing angle deposition (GLAD) or oblique angle deposition.

Atomistic methods are widely used for the simulation of normally deposited films and GLAD films. The effect of the deposition angle on the structure of hydrogenated amorphous silicon films was studied using the classical molecular dynamics (MD) simulation [5]. The horizontal dimensions of the simulation area were equal to $8.2 \mathrm{~nm}$ for both directions. The substrate contained approximately 9900 silicon atoms. The increase of the roughness and the formation of the slanted columnar structures with growth of the deposition angle were demonstrated. The effect of surface diffusion on the film structure was also investigated [5]. The structure of a growing film deposited by carbon atoms with an energy of $75 \mathrm{eV}$ was studied using the MD approach [6]. A $3.9 \mathrm{~nm}$ diamond slab, quadratic in the horizontal plane, was used as a substrate. Normal and near-normal deposition led to the formation of a film with a smooth surface. The increase of the deposition angle led to the increase of the roughness. MD 
simulation was also used to study the growth of diamond-like carbon films [7]. The MD simulation showed that an increase of the deposition angle results in the increase of the roughness and porosity. The residual stress was reduced significantly when the deposition angle reached $45^{\circ}$. The decrease of the compressive stress was caused by the relaxation of the distorted C-C bonds [7]. The MD and kinetic Monte Carlo methods were used to simulate growth of $\mathrm{TiO}_{2}$ films [8]. The formation of the columnar structure was demonstrated in the case of oblique angle deposition. The anisotropy of the refractive index was studied in [8], using the anisotropic Bruggeman effective medium theory for many-component materials [9]. The growth of microcolumns in the GLAD films was investigated using the 3D ballistic simulation $[10,11]$. Dependence of roughness on the deposition conditions of the titanium GLAD films was studied using MD simulation in [12].

In the present work, the dependence of the stress tensor components on the film thickness for high-energy-deposited silicon dioxide films is studied in the frame of the method developed in [13-15].

The procedure of stress calculations was proposed in [14], where it was applied to the normally deposited silicon dioxide films. In the present work, the developed procedure is applied for studying stresses in the films growing under different deposition angles, including GLAD films. These films exhibit markedly different structural properties compared to normally deposited films, and for this reason it should be expected that stresses in these films are also very different. In addition, stress distributions over the film thickness are calculated by the method described in [2]. Calculation of stresses even for the initial stage of film growth requires the simulation of atomistic clusters consisting of hundreds of thousands of atoms. The use of high-performance parallel computations for the first time allows the systematic full-atomistic simulations of such clusters and the calculation of stresses in silicon dioxide films at the initial stages of their growth.

The relationships between the deposition angle, film thickness, and stress values obtained are compared with experimental results.

\section{Method}

The deposition of $\mathrm{SiO}_{2}$ films was simulated using the MD-based procedure consisting of a given number of injection steps [13-15]. At each injection step, silicon and oxygen atoms with a stoichiometric proportion of 1:2 were inserted randomly at the top of the simulation box, indicated as "gas phase" in Figure 1. The empirical pairwise DESIL (DEposited films of SILica) force field was used for calculation of interatomic energy [13-15]. In the real chamber, we had $\mathrm{Si}$ atoms and $\mathrm{O}_{2}$ molecules. In the frame of our modeling approach, the oxygen was considered in the atomic form. The reason is as follows. In MD modeling, we take into account the final stage of the processes resulting in the formation of the film. At this stage, the silicon and oxygen atoms incoming from the gas phase interact with each other and with the surface atoms of the film. These interactions form covalent bonds, which leads to film growth.

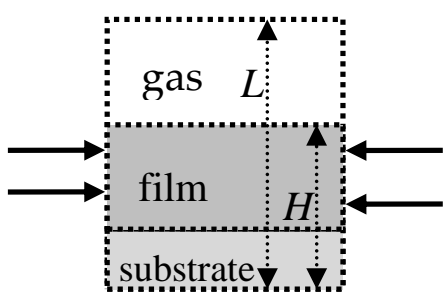

simulation box

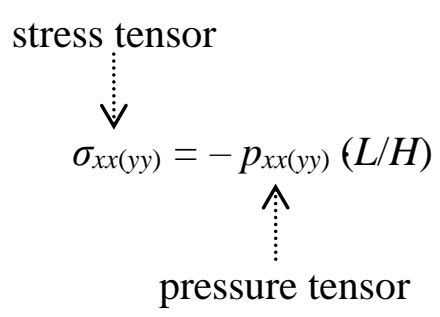

from molecular dynamics simulation

Figure 1. Calculation of the stress tensor components. Solid black arrows indicate external forces applied to keep the volume constant in the case of compressive stress. $L$ and $H$ are the vertical dimensions of the simulation box and film thickness, respectively. 
The specified stoichiometric proportion of silicon and oxygen atoms does not limit the formation of covalent bonds at the film surface. Atoms that have not formed these bonds were removed from the simulation box at the end of each injection cycle.

The total number of silicon and oxygen atoms per step was equal to 180 . This ensured the same flux density of deposited atoms as in previous works [13-15]. The initial values of the silicon and oxygen atom velocities corresponded to the deposited atoms' kinetic energies $E(\mathrm{Si})=10 \mathrm{eV}$ and $E(\mathrm{O})=0.1 \mathrm{eV}$. These values are typical for the high-energy deposition processes like ion beam sputtering (IBS) [16] and were used in MD simulations reported earlier [8,13-15].

At each injection step, the NVT (constant number of particles, volume, and temperature) ensemble with the periodic boundary conditions was used. The vertical dimension of the simulation box was increased by $\Delta h=0.01 \mathrm{~nm}$ at each injection step in order to compensate for the growth of film thickness. This $\Delta h$ value was calculated by taking into account that the average increase of the film thickness was equal to $2.5 \mathrm{~nm}$ at 250 injection steps. The horizontal dimensions of the box remained unchanged. The Berendsen thermostat [17] was applied to keep the simulation cluster temperature, $T=300 \mathrm{~K}$, constant. The duration of one injection step was $6 \mathrm{ps}$ and the time step of MD modeling was $0.5 \mathrm{fs}$.

Calculation of the stress dependence on the film thickness for different deposition angles was organized as follows:

1. Preparation of the fused silica substrate by the melting-quenching procedure from an alpha-quartz crystal [14]. Horizontal dimensions of substrate clusters were equal to 10 and $30 \mathrm{~nm}$, the vertical dimension was equal to $6 \mathrm{~nm}$. Horizontal dimensions of the cluster in the simulation of film deposition were the same as those of the substrate.

2. Choice of the deposition angle.

3. Simulation of the silicon dioxide film deposition as described above. The number of injection steps was $N_{s}=250$. This ensured the growth of layer with a thickness of approximately $2.5 \mathrm{~nm}$. During the simulation, it was revealed that at the initial stage of the film growth, the absolute value of the stress rapidly increased with increasing film thickness. To take this into account, $N_{S}$ was reduced to 100 if the film thickness was less than $10 \mathrm{~nm}$.

4. Simulation of the deposited cluster in the NVT ensemble and calculation of the pressure tensor components $p_{x x(y y)}$. The initial state for the simulation was the final state after the completion of $N_{s}$ injection steps. The length of the MD trajectory for averaging of $p_{x x(y y)}$ values was taken equal to 100 ps [15]. The stress tensor components were calculated as shown in Figure 1, where $L$ and $H$ are the vertical dimensions of the simulation box and film thickness, respectively. The multiplier $(L / H)$ in the equation of Figure 1 ensured the correction of pressure to the empty volume at the top part of the box [18].

5. Stages 3-4 were repeated until the film thickness achieved a specified value of about $40 \mathrm{~nm}$. This required approximately 4000 injection steps. The initial state for the simulation was the final state of stage 4 . The total number of atoms in the final cluster was about one million.

MD simulation was performed using the GROMACS package [19]. Components of the pressure tensor were calculated as follows [19]:

$$
\mathbf{p}=\frac{2}{V}(\mathbf{K}-\mathbf{\Xi})
$$

where $V$ is the volume of the simulation box, $\mathbf{K}$ and $\Xi$ are the kinetic energy and virial tensors, respectively:

$$
\mathbf{K}=\frac{1}{2} \sum_{i=1}^{N} m_{i} \vec{v}_{i} \otimes \vec{v}_{i} ; \quad \Xi=-\frac{1}{2} \sum_{i, j=1, i \neq j}^{N} \vec{f}_{i j} \otimes \vec{r}_{i j}
$$

Here, $\otimes$ means the direct product of two vectors. The same expression was used for the pressure and stress calculations in MD modeling in [20]. 
In the above scheme, the simulation with constant volume in step 4 takes into account the effect of the substrate on the film. Maintaining a constant volume leads to the appearance of stresses in the deposited films. These stresses were compensated by the external pressure applied to the boundaries of the simulation box, perpendicular to the substrate plane. Values of the pressure tensor components were not specified "by hand" but were calculated using Equations (1) and (2) from MD trajectories. Thus, the horizontal stress tensor components can be defined through the corresponding pressure tensor components. In the vertical direction, stress was absent since the gas phase (Figure 1) did not prevent the film expansion.

For visual analysis of the atomistic structures, the visual molecular dynamic (VMD) package was used [21]. The simulation was carried out using equipment at the shared research facilities of the high performance computing resources at Lomonosov Moscow State University [22].

\section{Results and Discussion}

Initial values of the deposited Si atom's velocities were specified as in our previous work [23]:

$$
v_{z}=-v_{0} \cos \alpha ; v_{\mathrm{x}}=v_{0} \sin \alpha ; v_{y}=0
$$

where $\alpha$ is the deposition angle (see Figure 2, left side) and $v_{0}$ corresponds to the initial kinetic energy of $\mathrm{Si}$ atoms, $E(\mathrm{Si})=10 \mathrm{eV}$. The variation in velocity directions of oxygen atoms did not noticeably affect the properties of the deposited films. For this reason, the initial velocities of oxygen atoms were directed to the substrate perpendicular to its surface.

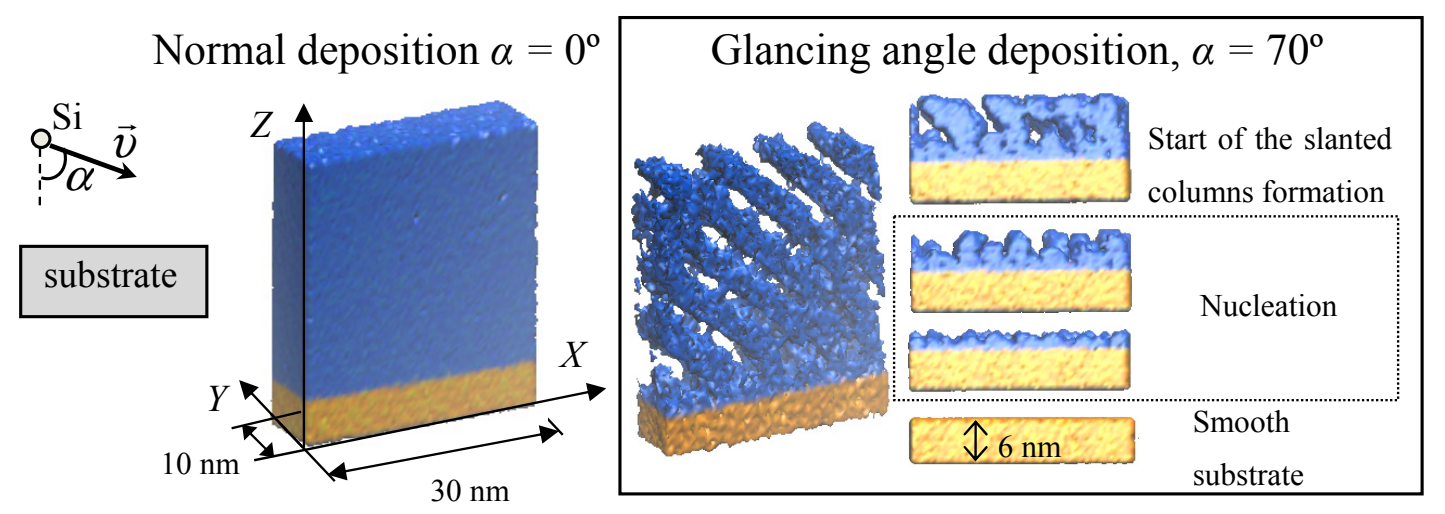

Figure 2. Structure of normally deposited and glancing angle deposited films.

The differences in the structure of normally deposited films and GLAD films are shown in Figure 2. In GLAD films, the flux fluctuations of incoming atoms resulted in the density fluctuations of the substrate surface. Due to the shadow effect [3], these fluctuations became the nuclei of future slanted columns (Figure 2, right side). The columnar structures were formed when the thickness of the film exceeded a few nanometers. The voids between the columns are a characteristic feature of the GLAD films [3]. These voids lead to a decrease in the density and refractive index of GLAD films compared with corresponding values for the normally deposited films.

The stress tensor components were calculated for both normally deposited films and GLAD films.

Results are shown in Figure 3. A negative sign of the $\sigma_{x x(y y)}$ values means that the stress is compressive. Values of the off-diagonal components $\sigma_{x y}=\sigma_{y x}$ and values of the root mean square deviation of the $\sigma$ are approximately several MPa. Thickness of the transition layer between substrate and film was about of $10 \mathrm{~nm}$ for the case of the normal deposition (top row in Figure 3). Since the substrate was prepared using the NPT ensemble under the pressure of $P=1 \mathrm{~atm}$ [14], the values of the stress tensor components are close to zero for $H$ values smaller than the substrate thickness. 

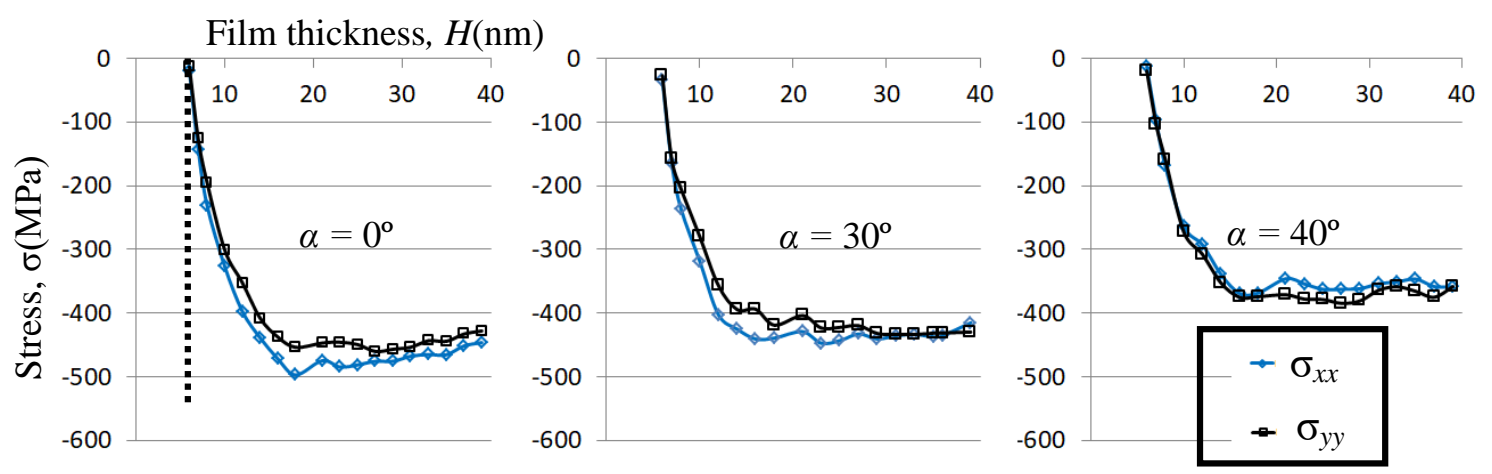

Film thickness, $H(\mathrm{~nm})$

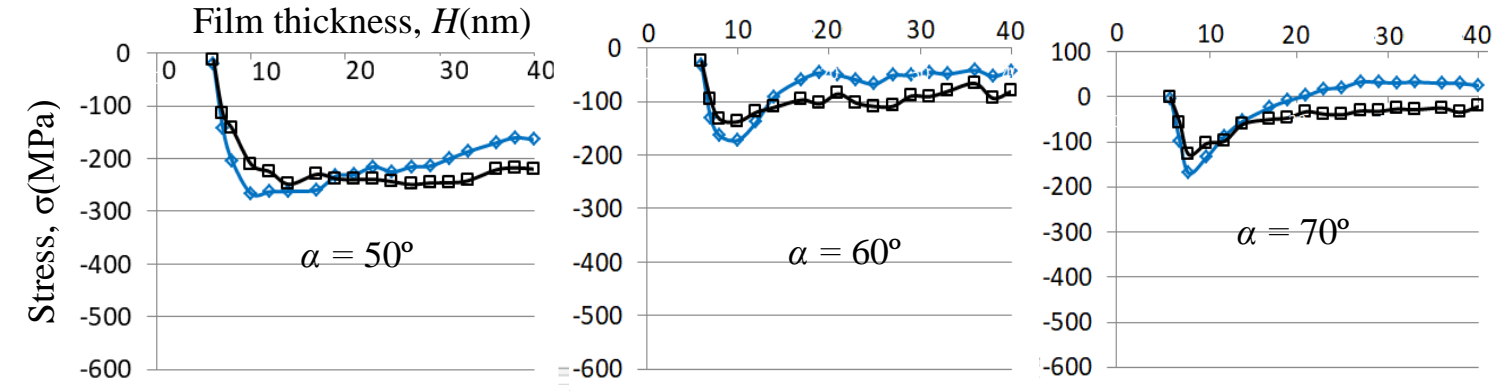

Figure 3. Dependencies of the stress tensor components $\sigma_{x x(y y)}$ on film thickness for different values of the deposition angle $\alpha$. Vertical dotted line indicates the substrate boundary. Axis orientations are shown in Figure 2.

For all of the deposition angles, the initial stage of the film growth was accompanied by a sharp increase in the absolute values of $\sigma_{x x(y y)}$. This is because the high-energy silicon atoms push the substrate material away from the point of impact (black arrows in Figure 4), which results in the increase of the pressure and stresses at the boundaries of the simulation box. Since the deposition started from the same substrate for all deposition angles, this effect appeared both for the normal deposition and for the glancing angle deposition. The resulting pressure growth correlated with a local densification of the GLAD films in the layer closest to the substrate. Density increased from the substrate value $2.2 \mathrm{~g} / \mathrm{cm}^{3}$ to approximately $2.4 \mathrm{~g} / \mathrm{cm}^{3}$, which corresponded to the density of the normally deposited films, and then decreased to a value of approximately $1.5 \mathrm{~g} / \mathrm{cm}^{3}$, corresponding to the averaged density of GLAD films.

normal deposition
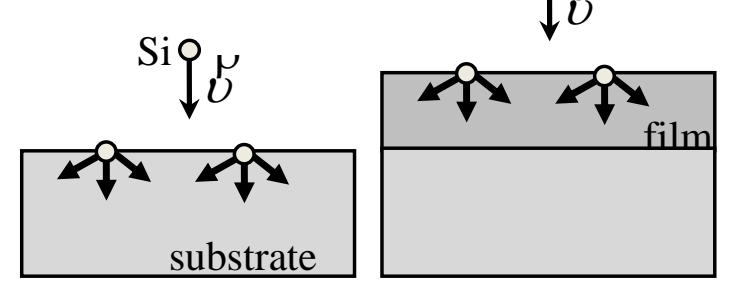

Figure 4. Explanation for the differences in the stress dependence on film thickness for normal deposition and glancing angle deposition (GLAD). See the text for the details.

With increase of the film thickness, a dependence of the structure on the deposition angle appeared. High-energy normal deposition produced a dense film and the local pressure appearing around the 
impact point was transmitted to the boundaries of the simulation box, just as in the initial stage of film growth. In contrast, glancing angle deposition resulted in the formation of a highly porous structure consisting of separate slanted columns (Figure 4, right side). In this case, the pressure appearing around the impact point could be absorbed by voids between the columns. This is why the pressure transmitted to the boundaries was significantly lower than in the case of a normal deposition.

The described effect resulted in the differences of stress behavior for normal and GLAD films when film thickness $H$ exceeded approximately ten nanometers. For the case of normal deposition, absolute values of $\sigma_{x x(y y)}$ slightly decreased with growth of $H$ (top row in Figure 3). The relative difference of stress tensor components $\left|\sigma_{x x}-\sigma_{y y}\right| /\left|\sigma_{x x(y y)}\right|<<1$ for the cases of $\alpha=0^{\circ}, 30^{\circ}, 40^{\circ}$. In contrast, in GLAD films $\left(\alpha=60^{\circ}, 70^{\circ}\right)$, values of $\left|\sigma_{x x(y y)}\right|$ were essentially reduced with growth of film thickness. The parameter $\left|\sigma_{x x}-\sigma_{y y}\right| /\left|\sigma_{x x(y y)}\right|$, characterizing the stress anisotropy, increased with growth of $H$. It is explained by the anisotropy of the GLAD film's structure in horizontal directions (right structure in Figure 2). In the case of $\alpha=50^{\circ}, \sigma(H)$ dependencies show the intermediate behavior between normal deposition and glancing angle deposition.

The effect of substrate dimensions to stresses was also studied. Values of stresses in the films deposited under angles $\alpha=0^{\circ}$ and $70^{\circ}$ on the substrate with horizontal dimensions of $20 \times 60 \mathrm{~nm}$ and vertical dimension $6 \mathrm{~nm}$ were calculated. The corresponding atomistic clusters were obtained in our previous work [23]. The deposition parameters, excluding cluster dimensions, were the same as those described in Section 2. In the case of normal deposition $\left(\alpha=0^{\circ}\right)$, relative difference in stress values due to the difference in substrate dimensions was about $10 \%$. In GLAD film $\left(\alpha=70^{\circ}\right)$, the maximum absolute stress values corresponded to a film thickness of about $10 \mathrm{~nm}$ and exceeded by approximately 1.5 times the stress values obtained for the same thickness of the film deposited on the $10 \mathrm{~nm} \times 30 \mathrm{~nm}$ substrate (bottom row in Figure 3). With growth of film thickness, the stress was reduced to almost the same value as shown in the right plots of the bottom row of Figure 3.

Stresses were also calculated for the structures obtained in MD simulations of GLAD with the alternation of deposition angle and with substrate rotation at fixed $\alpha$ [24]. The alternation means that the $v_{\mathrm{x}}$ in Equation (3) periodically changes sign. The rotation means that velocity components in Equation (3) change with time $t$ as follows: $v_{\mathrm{x}}=v_{0} \operatorname{sn}(\alpha) \operatorname{sn}(\omega t)$ and $v_{\mathrm{y}}=v_{0} \operatorname{sn}(\alpha) \cos (\omega t)$, where $\omega$ is rotation speed [24]. The type of growing-film structure, chevron-like or tree-like, depended on the relationships between the alternation period and the rate of film growth. Chevron-like structures and tree-like structures were characterized by compressive stress values of about $-(20 \div 40) \mathrm{MPa}$. In contrast, the tensile stress of about $20 \mathrm{MPa}$ was obtained for the helical structure.

The stress distribution functions $\sigma_{h \_x x(y y)}(h)$ along the vertical coordinate $h$ were determined through the derivative of the product of averaged stresses $\sigma_{x x(y y)}$ and film thickness with respect to the vertical coordinate [2]. We assumed that the stress does not change after deposition. The calculated values of $\sigma_{h \_x x(y y)}(h)$ were averaged over an interval of $10 \mathrm{~nm}$ to smooth out fluctuations arising from differentiation. Results are presented in Figure 5. Stress components in the normally deposited films were compressive for all values of vertical coordinate. Their absolute values decreased when the value of $h$ exceeded $20 \mathrm{~nm}$. For GLAD film, stresses were compressive when the vertical coordinate $h$ was less than $15 \mathrm{~nm}$. Then the stress changed sign and became tensile. The absolute values of $\sigma_{h \_} x x(y y)(h)$ decreased with increasing vertical coordinate when $h$ exceeded $20 \mathrm{~nm}$. 

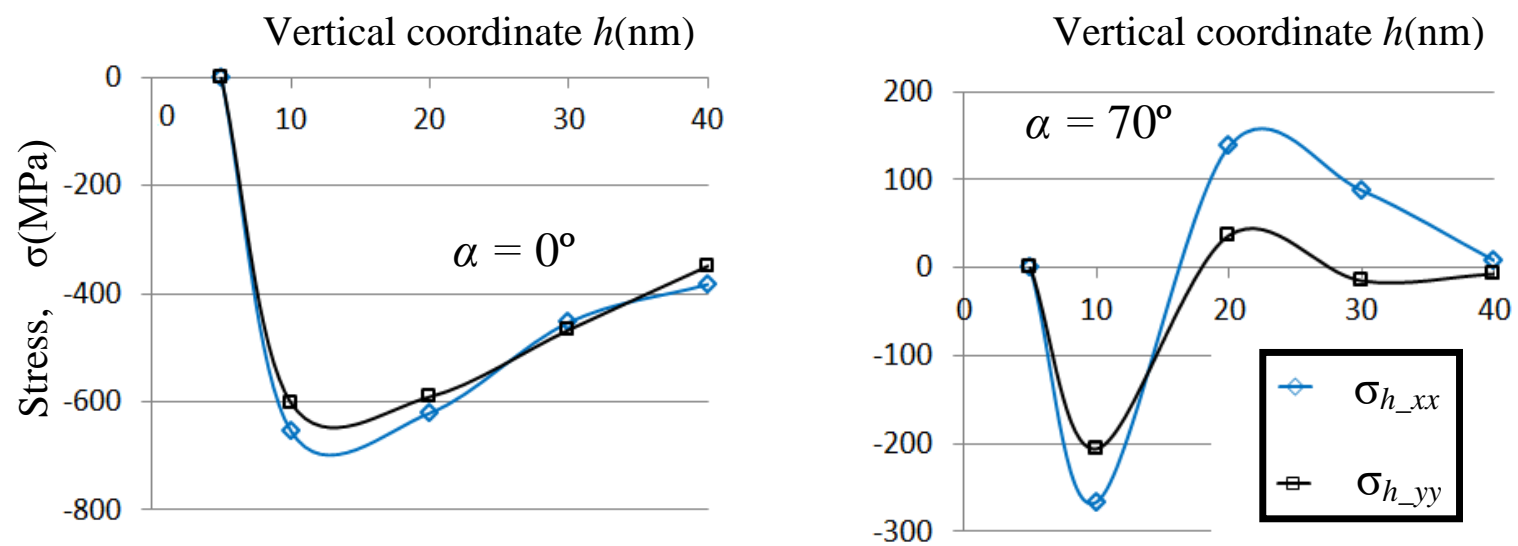

Figure 5. Distribution of the stress tensor components over the vertical coordinate $h, \alpha$ is the deposition angle.

Our results of the stress calculations were compared with the available experimental data obtained by other research groups. They found a decrease in stress values with increasing deposition angle (Figure 3), which was previously reported for different films deposited by magnetron sputtering [25]. The absolute value of the compressive stress in a normally deposited film (upper row in Figure 3) was in the range of experimental data $[1,2,26]$. The change of stress from compressive to tensile with the growth of deposition angle was observed experimentally for the silicon dioxide films fabricated by electron beam evaporation [27]. Such a change was detected in our simulation (see results for $\alpha=70^{\circ}$ in Figure 3). Since the electron beam evaporation is a low-energetic process, the experimental stress values in [27] were lower than those obtained in our simulation. A change in the sign of stress with an increase in the deposition angle was also found for GLAD diamond-like carbon films [7] and hafnium nitride films [28]. Thus, the simulation results we obtained are in good qualitative agreement with previously reported experimental results.

The dependencies of stress, density, and refractive index on the deposition angle are shown in Figure 6. These dependencies were obtained by averaging of the respective parameters over the volume, from $H=30 \mathrm{~nm}$ to $H=40 \mathrm{~nm}$.
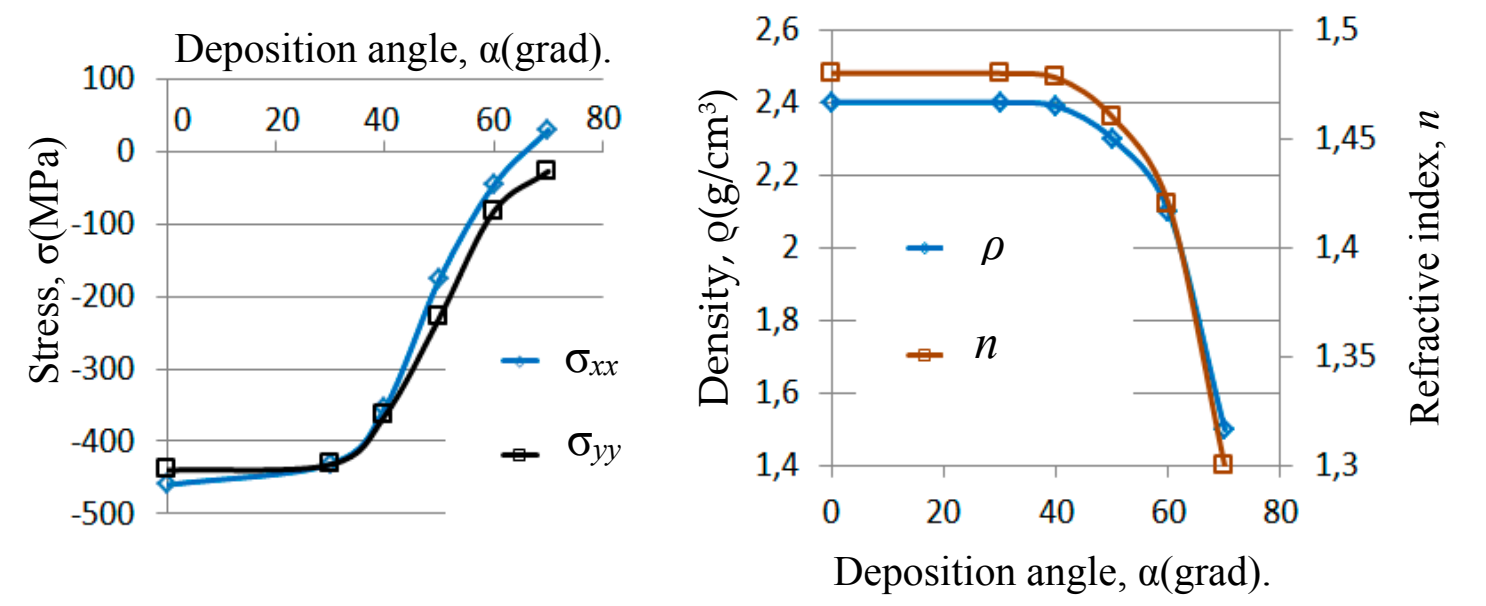

Figure 6. Dependences of the stress tensor components, film density, and refractive index on the deposition angle.

Refractive index $n$ was calculated using the Maxwell relation between refractive index and dielectric constant $n^{2}=\varepsilon$ and Bruggeman effective medium theory [29] for the porous films [9]:

$$
\left(1-f_{1}\right)\left(\varepsilon_{1}-\varepsilon\right) /\left(\varepsilon_{1}+2 \varepsilon\right)+f_{1}(1-\varepsilon) /(1+2 \varepsilon)=0
$$


where $f_{1}$ is the void volume fraction, $\varepsilon_{1}$ is the dielectric constant of dense film fraction, $\varepsilon$ is the effective dielectric constant. Refractive index of dense silicon dioxide film deposited on fused silica by the IBS process is equal to $1.48(632 \mathrm{~nm})$ [30], so $\varepsilon_{1}=2.19$. Dependence of $f_{1}$ on the deposition angle $\alpha$ is defined as follows:

$$
\mathrm{f} 1(\alpha)=\mathrm{V} 1(\alpha) / \mathrm{V}(\alpha)=1-\rho(\alpha) / \rho(0)
$$

where $V_{1}(\alpha)$ and $V(\alpha)$ are the void volume and the total film volume, respectively, $\rho(\alpha)$ is the film density averaged over the volume from $H=30 \mathrm{~nm}$ to $H=40 \mathrm{~nm}, \rho(0)=2.4 \mathrm{~g} / \mathrm{cm}^{3}$ is the density of the normally deposited film. It was assumed that the normally deposited film has no free volume and the density inside the slanted columns of GLAD films was equal to $\rho(0)$. Refractive index was calculated as $n=\sqrt{ } \varepsilon$ and its dependence on $\alpha$ was found by using Equations (4) and (5).

As expected, the refractive index decreased with growth of the deposition angle, which correlates with the decrease of the stress tensor components. The density dependence on the deposition angle agreed with the earlier reported results of the atomistic simulation using the ballistic approach [11]. The films with low refractive index, $n=1.32\left(\alpha=70^{\circ}\right)$, were characterized by low values of both stress tensor components. Values of the refractive index for large deposition angles were in the interval of the experimental results for $\mathrm{SiO}_{2}$ GLAD films [31].

\section{Conclusions}

The molecular dynamics study of stresses in growing silicon dioxide film for the different values of deposition angle was performed. The stress tensor components were calculated through the pressure tensor.

The stresses were compressive and varied slightly with the deposition angle growth from $0^{\circ}$ to approximately $30^{\circ}$ in the all investigated intervals of film thickness. A further increase of the deposition angle led to a decrease in the absolute value of stresses. Stresses in GLAD films were mainly compressive and their values were several times smaller than in the case of normally deposited films. The anisotropy of stresses in the substrate plane was revealed for GLAD films. The decrease of the stresses correlated with the decrease of the film density and refractive index.

This work was devoted to the study of stresses arising at the initial stage of growth of silicon dioxide films. In experiments, stresses are usually determined for films having thicknesses more than 40-50 nm [1]. Thus, simulations can supplement experimental data in the range of small thicknesses.

For GLAD films, the results obtained demonstrate stress changes that accompany the transition of film growth from the nucleation stage to the formation of slanted columns.

The method developed in the present work can be used to study stresses in other oxide films.

Author Contributions: Conceptualization, A.V.T. and V.B.S.; methodology, F.V.G.; software, F.V.G.; validation, A.V.T., V.B.S., F.V.G.; formal analysis, F.V.G.; investigation, F.V.G.; resources, A.V.T., V.B.S.; data curation, F.V.G.; writing - F.V.G.; writing - A.V.T., V.B.S.; visualization, F.V.G.; supervision, A.V.T.; project administration, A.V.T.; funding acquisition, A.V.T. All authors have read and agreed to the published version of the manuscript

Funding: This research was funded by Russian Science Foundation, grant number 19-11-00053.

Acknowledgments: The work was supported by the Russian Science Foundation (grant number 19-11-00053). The work was carried out according to the research program of Moscow Center of Fundamental and Applied Mathematics.

Conflicts of Interest: The authors declare no conflict of interest.

\section{References}

1. Begou, T.; Lumeau, J. Accurate analysis of mechanical stress in dielectric multilayers. Opt. Lett. 2017, 42, 3217-3220. [CrossRef]

2. Abadias, G.; Chason, E.; Keckes, J.; Sebastiani, M.; Thompson, G.B.; Barthel, E.; Doll, G.L.; Murray, C.E.; Stoessel, C.H.; Martinu, L. Review Article: Stress in thin films and coatings: Current status, challenges, and prospects. J. Vac. Sci. Tech. A 2018, 36, 020801. [CrossRef] 
3. Hawkeye, M.M.; Brett, M.J. Glancing angle deposition: Fabrication, properties, and applications of microand nanostructured thin films. J. Vac. Sci. Technol. 2007, 25, 1317-1335. [CrossRef]

4. Robbie, K.; Brett, M.J.; Lakhtakia, A. Chiral sculptured thin films. Nature 1996, 384, 616. [CrossRef]

5. Luo, Y.; Lin, M.; Zhou, N.; Huang, H.; Tsai, C.-T.; Zhou, L. Molecular dynamics simulation study of the microstructure of a-Si:H thin film grown by oblique-angle deposition. Phys. B Condens. Matter 2018, 545, 80-85. [CrossRef]

6. Joe, M.; Moon, M.-W.; Oh, J.; Lee, K.-H.; Lee, K.-R. Molecular dynamics simulation study of the growth of a rough amorphous carbon film by the grazing incidence of energetic carbon atoms. Carbon 2012, 50, 404-410. [CrossRef]

7. Li, X.; Ke, P.; Lee, K.-R.; Wang, A. Molecular dynamics simulation for the influence of incident angles of energetic carbon atoms on the structure and properties of diamond-like carbon films. Thin Solid Films 2014, 552, 136-140. [CrossRef]

8. Badorreck, H.; Steinecke, M.; Jensen, L.; Ristau, D.; Jupé, M.; Müller, J.; Tonneau, R.; Moskovkin, P.; Lucas, S.; Pflug, A.; et al. Correlation of structural and optical properties using virtual materials analysis. Opt. Express 2019, 27, 22209-22225. [CrossRef] [PubMed]

9. Schmidt, D.; Schubert, M. Anisotropic Bruggeman effective medium approaches for slanted columnar thin films. J. Appl. Phys. 2013, 114, 083510. [CrossRef]

10. Smy, T.; Vick, D.; Brett, M.J.; Dew, S.K.; Wu, A.T.; Sit, J.C.; Harris, K.D. Three-dimensional simulation of film microstructure produced by glancing angle deposition. J. Vac. Sci. Technol. A 2000, 18, 2507-2512. [CrossRef]

11. Grüner, C.; Liedtke, S.; Bauer, J.; Mayr, S.G.; Rauschenbach, B. Morphology of Thin Films Formed by Oblique Physical Vapor Deposition. ACS Appl. Nano Mat. 2018, 1, 1370-1376. [CrossRef]

12. Backholm, M.; Foss, M.; Nordlund, K. Roughness of glancing angle deposited titanium thin films: An experimental and computational study. Nanotechnology 2012, 23, 385708. [CrossRef] [PubMed]

13. Grigoriev, F.V.; Sulimov, A.V.; Kochikov, I.V.; Kondakova, O.A.; Sulimov, V.B.; Tikhonravov, A.V. Supercomputer Modeling of the ion Beam Sputtering Process: Full-Atomistic Level. In Proceedings of the Optical Systems Design 2015: Advances in Optical Thin Films V, Jena, Germany, 7-10 September 2015; International Society for Optics and Photonics: Bellingham, WA, USA, 2015; Volume 9627, p. 962708. [CrossRef]

14. Grigoriev, F.V.; Sulimov, A.V.; Katkova, E.V.; Kochikov, I.V.; Kondakova, O.A.; Sulimov, V.B.; Tikhonravov, A.V. Computational Experiments on Atomistic Modeling of Thin Film Deposition. Appl. Opt. 2017, 56, C87-C90. [CrossRef] [PubMed]

15. Grigoriev, F.V.; Sulimov, A.V.; Katkova, E.V.; Kochikov, I.V.; Kondakova, O.A.; Sulimov, V.B.; Tikhonravov, A.V. Annealing of deposited $\mathrm{SiO}_{2}$ thin films: Full-atomistic simulation results. Opt. Mat. Express 2016, 6, 3960-3966. [CrossRef]

16. Tabata, A.; Matsuno, N.; Suzuoki, Y.; Mizutani, T. Oprtical properties and structure of SiO2 films prepared by ion-beam sputtering. Thin Solid Films 1996, 289, 84-89. [CrossRef]

17. Berendsen, H.J.C.; Postma, J.P.M.; van Gunsteren, W.F.; DiNola, A.; Haak, J.R. Molecular-Dynamics with Coupling to an External Bath. J. Chem. Phys. 1984, 81, 3684-3690. [CrossRef]

18. Zheng, H. Molecular Dynamic Simulation of Thin Film Growth Stress Evolution. Ph.D. Thesis, Lehigh University, Bethlehem, PA, USA, September 2011.

19. Abraham, M.J.; Murtola, T.; Schulz, R.; Páll, S.; Smith, J.C.; Hess, B.; Lindahl, E. GROMACS: High performance molecular simulations through multi-level parallelism from laptops to supercomputers. SoftwareX 2015, 1, 19-25. [CrossRef]

20. Al, M.; Webb III, E.B. A case study of thin film stress evolution at a dissimilar material interface via molecular dynamics simulations: Gold film growth on a nickel substrate. Nanomater. Nanotechnol. 2018, 8, 1-12. [CrossRef]

21. Humphrey, W.; Dalke, A.; Schulten, K. VMD: Visual molecular dynamics. J. Mol. Gr. 1996, 14, 33-38. [CrossRef]

22. Voevodin, V.V.; Antonov, A.S.; Nikitenko, D.A.; Shvets, P.A.; Sobolev, S.I.; Sidorov, I.Y.; Stefanov, K.S.; Voevodin, V.V.; Zhumatiy, S.A. Supercomputer Lomonosov-2: Large Scale, Deep Monitoring and Fine Analytics for the User Community. Supercomput. Front. Innov. 2019, 6, 4-11. [CrossRef]

23. Grigoriev, F.V.; Sulimov, V.B.; Tikhonravov, A.V. Atomistic simulation of the glancing angle deposition of $\mathrm{SiO} 2$ thin films. J. Non Cryst. Solids 2019, 512, 98-102. [CrossRef] 
24. Grigoriev, F.V.; Sulimov, V.B.; Tikhonravov, A.V. Structure of Highly Porous Silicon Dioxide Thin Film: Results of Atomistic Simulation. Coatings 2019, 9, 568. [CrossRef]

25. Siad, A.; Besnard, A.; Nouveau, C.; Jacquet, P. Critical angles in DC magnetron glad thin films. Vacuum 2016, 131, 305-311. [CrossRef]

26. Bischoff, M.; Nowitzki, T.; Voß, O.; Wilbrandt, S.; Stenzel, O. Postdeposition treatment of IBS coatings for UV applications with optimized thin-film stress properties. Appl. Opt. 2014, 53, A212-A220. [CrossRef] [PubMed]

27. Tolenis, T.; Grineviciute, L.; Buzelis, R.; Smalakys, L.; Pupka, E.; Melnikas, S.; Selskis, A.; Drazdys, R.; Melninkaitis, A. Sculptured anti-reflection coatings for high power lasers. Opt. Mater. Express 2017, 7, 1249-1258. [CrossRef]

28. Abadias, G.; Angay, F.; Mareus, R.; Mastail, C. Texture and Stress Evolution in HfN Films Sputter-Deposited at Oblique Angle. Coatings 2019, 9, 712. [CrossRef]

29. Stroud, D. Generalized effective-medium approach to the conductivity of an inhomogeneous material. Phys. Rev. B 1975, 12, 3368. [CrossRef]

30. Jiang, Y.; Liu, H.; Wang, L.; Liu, D.; Jiang, C.; Cheng, X.; Yang, Y.; Ji, Y. Optical and interfacial layer properties of $\mathrm{SiO} 2$ films deposited on different substrate. Appl. Opt. 2014, 53, A83-A87. [CrossRef]

31. Melninkaitis, A.; Grinevičiūtè, L.; Abromavičius, G.; Mažulè, L.; Smalakys, L.; Pupka, E.; Ščiuka, M.; Buzelis, R.; Kičas, S.; Tolenis, T. Next-generation allsilica coatings for UV applications. In Proceedings of the Laser-Induced Damage in Optical Materials 2017, Boulder, CO, USA, 24-27 September 2017; International Society for Optics and Photonics: Bellingham, WA, USA. [CrossRef]

(C) 2020 by the authors. Licensee MDPI, Basel, Switzerland. This article is an open access article distributed under the terms and conditions of the Creative Commons Attribution (CC BY) license (http://creativecommons.org/licenses/by/4.0/). 